\title{
Evelyne GAGNON
}

\section{Pour une définition de la poésie fantastique}

Dans la foulée de son étude intitulée Le Fantastique (Flammarion, 2006), Michel Viegnes publie un second ouvrage sur cette catégorie esthétique. L'Envoûtante étrangeté. Le fantastique dans la poésie française (1820-1924) s'adresse à un lectorat d'initiés, plongeant plus avant dans les questions de la poésie et du fantastique. Circonscrivant cette fois son étude autour d'un corpus poétique ciblé — alors que son ouvrage précédent s'intéressait tant à la poésie qu'au roman, au cinéma et à l'image, confrontant des auteurs d'époques et de nationalités différentes —, Viegnes se propose de considérer la poésie fantastique en tant que sous-ensemble de la poésie comme genre.

D'entrée de jeu, l'auteur annonce son entreprise :

l'intérêt véritable d'une théorie de la poésie fantastique n'est pas de juxtaposer deux grandes constructions théoriques, pour y chercher, et y déceler sans doute, des passerelles, voire des briques communes. Le postulat de départ, ici, est qu'un effort pour définir une poésie fantastique sera une contribution à l'effort de définition de la poésie et du fantastique. (p. 8)

Viegnes s'intéresse à identifier la spécificité du fantastique dans la poésie française, du romantisme à l'orée du surréalisme et, ce faisant, à définir dans une certaine mesure la poésie et le fantastique, l'association des deux termes opérant selon lui une révélation, alors que « [c]hacun contient, dans ses profondeurs sémantiques, une clé du domaine de l'autre» (ibid.). L'une de ces clés révélatrices, qui ouvre la porte à l'ensemble de l'étude, se trouve dans une visée propre à la poésie et au fantastique. Poésie et fantastique ont pour fonction commune d'inquiéter, de perturber le sens des choses, de remettre en cause les paradigmes sans s'éloigner totalement du réel. Leur quête de transfiguration, leur " tentative décalée de réenchantement du monde » (p. 13) n'est pas sans laisser une part d'ombre, d'inexpliqué, qui bouleverse la lecture : 
poésie et fantastique ne sont ni désolidarisés du monde référentiel, sous peine de tomber dans l'affabulation ludique ou le merveilleux pur — ce que Caillois appelle la féerie — ni complices de l'illusion collective de la vie ordinaire. Ils sont, comme la Vénus d'Ille, des éléments de «turbulence » qui visent à troubler, à désordonner, à reconfigurer notre relation symbiotique avec les mots et les choses. «Belle fonction», disait Gide, «que celle d'inquiéteur»: poésie et fantastique procèdent de ce principe d'inquiétude, qui ne se confond pas nécessairement avec la peur. (p. 9-10)

Nous retrouvons dans ce point de convergence entre poésie et fantastique l'hypothèse majeure que défendra Viegnes au fil de son ouvrage, hypothèse qui renouvelle les travaux sur le fantastique. Comme son titre l'indique, le fantastique en poésie n'est pas «inquiétante étrangeté », expression de Freud reprise par les théoriciens, mais «envoûtante étrangeté ». Paradoxalement, l'inquiétude créée par le fantastique est positive en poésie; elle ne vise pas à terrifier, mais assume une fonction ambivalente : elle inquiète en même temps qu'elle exalte. Cette hypothèse lancée, Viegnes poursuit une réflexion en trois temps: traçant d'abord un état présent de la recherche sur les théories du fantastique et du genre poétique, il construit son étude autour de certains thèmes de cette poésie fantastique, puis autour de certaines figures du langage, toutes aussi révélatrices de cet envoûtement.

Dans son premier chapitre, "Poésie fantastique et fantastique poétique », Viegnes fait le point sur les différentes théories au sujet du fantastique et de la poésie, et remet à l'ordre du jour certaines vérités. Ainsi, il rappelle que "[1] fantastique [...] n'est pas un genre mais une catégorie esthétique, au même titre que le grotesque, le tragique ou le comique » (p. 48). Si plusieurs théoriciens ont cru que le fantastique ne pouvait exister en-dehors du récit, le travail de Michel Viegnes consiste précisément à démanteler ce postulat, ce qui fait en grande partie l'intérêt de son étude. En prenant pour objet le fantastique dans la poésie, l'auteur s'inscrit à l'encontre de Tzvetan Todorov et d'Irène Bessières : le fantastique, selon Viegnes, n'est pas «intrinsèquement diégétique » (p. 45), il ne se réduit pas à un seul genre littéraire, mais dépasse les frontières génériques et se voit récupéré dans les variations infinies du langage et des représentations (dans la poésie, au cinéma, 
voire en musique ou dans d'autres formes de discours). C'est que l'effet fantastique ne peut s'objectiver et se réduire à un ensemble de procédés et de motifs, car il est le résultat d'une attitude particulière, d'un certain regard, "un prisme qui sert à interroger les limites du réel» (p. 48). Ainsi, le fantastique prend non seulement forme dans le récit linéaire, mais aussi dans la poésie, qu'il habite plus aisément: libéré des contraintes de la linéarité, le poème peut facilement entretenir un flou énigmatique, une béance du sens.

Les chapitres « Motifs » et «Langage » constituent le corps de l'étude. Dans le premier, Viegnes fait l'étude de motifs récurrents, invariants, du fantastique, qui hantent la poésie de son corpus (chimères, ombres, lieux, ténèbres, voix) et exemplifie chacun d'eux par l'analyse de poèmes choisis. Dans "Langage ", dernier chapitre de l'ouvrage, l'auteur adopte une formule analogue : à partir de trois constituants propres à la dimension sensible et matérielle du langage poétique (noms, métrique, transfigurations), l'auteur analyse plusieurs poèmes pour étoffer son propos. La forme du poème, ses figures rhétoriques, sa disposition, son rythme sont autant de traits du langage qui contribuent, comme les motifs, à l'avènement du fantastique : «[d]ans la poésie fantastique, le sentiment d'étrangeté ne peut être induit par les seuls outils que le récit utilise; interviennent également ces figures que l'œil déchiffre dans l'espace paginal et que l' "oreille absolue" traduit en échos » (p. 293). Bien que le choix des poètes soit riche et diversifié - les auteurs consacrés côtoient les minores - et que les figures relevées soient des plus pertinentes, le tout s'organise de façon répétitive, avec des analyses quelque peu rapides et cursives qui prennent bientôt la forme d'un inventaire plutôt que d'une étude suivie. Si les hypothèses lancées en début d'étude sont des plus intéressantes, malheureusement elles ne font que rester en germe dans ces deux chapitres d'analyses. Le fil est tout de même récupéré en conclusion. Viegnes rappelle que le fantastique en poésie fait rarement peur, qu'il remplit plutôt la fonction positive d'explorer les frontières de la raison. "Le propre de la poésie fantastique est de révéler une envôutante étrangeté, de distiller une inquiétude féconde, qui incite à donner tout son crédit au rêve, et à comprendre pourquoi la raison ne saurait avoir toujours raison [...] [E]n tant que quête des suppléments 
de sens, exploration des marges du dicible et vision transfiguratrice des choses, [la poésie] a devoir de cité dans le fantastique. » (p. 349)

Certes, cet ouvrage comporte quelques lacunes. Le premier chapitre, dans lequel l'auteur ourdit ses prémices théoriques, est quelque peu entravé par une exhaustivité qui alourdit inutilement le propos et crée un déséquilibre avec les chapitres suivants, dont les analyses textuelles manquent de profondeur. En outre, si l'auteur s'engage à définir la spécificité de la poésie du $\mathrm{XIX}^{\mathrm{e}}$ siècle, son étude repose sur une conception anhistorique de la poésie. Viegnes ouvre par le fait même à une relecture intéressante de la poésie en général, sous l'angle de l'inquiétude, mais n'apporte pas de contre-modèle ou de nuance à cette fonction.

Toutefois, l'étude a le mérite de remettre en question les acceptions du fantastique et de revisiter cette notion, de l'extraire de son contexte diégétique pour en faire une catégorie esthétique applicable à la poésie. Si une grande partie de ses réflexions tournent autour de Hugo, Baudelaire et Mallarmé, Viegnes nous surprend par les analyses qu'il fournit de poésies comme celles de Joséphin Péladan, de Xavier Forneret, de Louis Le Cardonnel et de bien d'autres. Dans sa démarche, l'auteur ne prend pas seulement en considération le point de vue des théoriciens, mais accorde une place privilégiée aux discours des praticiens, poètes et romancier. Finalement, les comparaisons avec le récit et le roman, qui se multiplient au fil de l'ouvrage, enrichissent la réflexion et élargissent les perspectives, conduisent le fantastique en terrain « transgénérique ».

Référence: Michel Viegnes, L'Envoûtante étrangeté. Le fantastique dans la poésie française (1820-1924), Grenoble, Presses universitaires de Grenoble, coll. «Bibliothèque de l'imaginaire », 2006, 375 p. 